\title{
Measurements of the top quark pair production cross section in pp collisions at $7 \mathrm{TeV}$ using the CMS detector
}

\author{
Andreas B. MEYER ${ }^{* \dagger}$ \\ DESY \\ E-mail: andreas.meyerecern.ch
}

\begin{abstract}
Measurements of the top-pair production cross section in proton-proton collisions at the CMS Experiment at the LHC are presented. The data were collected at a centre-of-mass energy of 7 $\mathrm{TeV}$. Measurements performed on an integrated luminosity of $36 \mathrm{pb}^{-1}$ of data collected in 2010 are presented in $t \bar{t}$ final states with one or two leptons (muon or electron) and jets. Moreover, first measurements of the cross section for the production of top quark pairs are presented in the fully hadronic channel, and in the dilepton final state with one muon and one tau-lepton. These measurements make use of $1.1 \mathrm{fb}^{-1}$ of data recorded in 2011.
\end{abstract}

XXIst International Europhysics Conference on High Energy Physics

21-27 July 2011

Grenoble, Rhône-Alpes France

*Speaker.

On behalf of the CMS Collaboration 


\section{Introduction}

The top quark is the heaviest elementary particle known to date, and as such it plays a special role in the electro-weak symmetry breaking as well as many models of New Physics beyond the Standard Model. At the LHC, top quarks are predominantly produced in pairs, through gluon-gluon fusion. Theory predictions performed to approximate next-to-next-to-leading order (NNLO*) predict an inclusive $t \bar{t}$ cross section of about $164 \pm 10 \mathrm{pb}[2,3]$ or $149 \pm 11 \mathrm{pb}$ [4]. Top quarks decay to nearly $100 \%$ into $W$-bosons and $b$-quarks. The presence of $b$-quarks is detected experimentally by their decay length, leading to a separation of tracks from the primary vertex. Experimentally, $t \bar{t}$ pair events are classified in the dileptonic, the lepton+jets and the fully hadronic channel, according to the decays of the two $W$-bosons, with branching ratios of $10 \%, 48 \%$ and $42 \%$ respectively. For all of these final states, except $e \tau$ and $\tau \tau$, cross section measurements have been performed at the CMS Experiment [1] and are presented here. Consistency between the measurements in different decay channels constitutes an important test of the Standard Model, as possible new physics may appear differently in the different channels.

\section{Dilepton Channel}
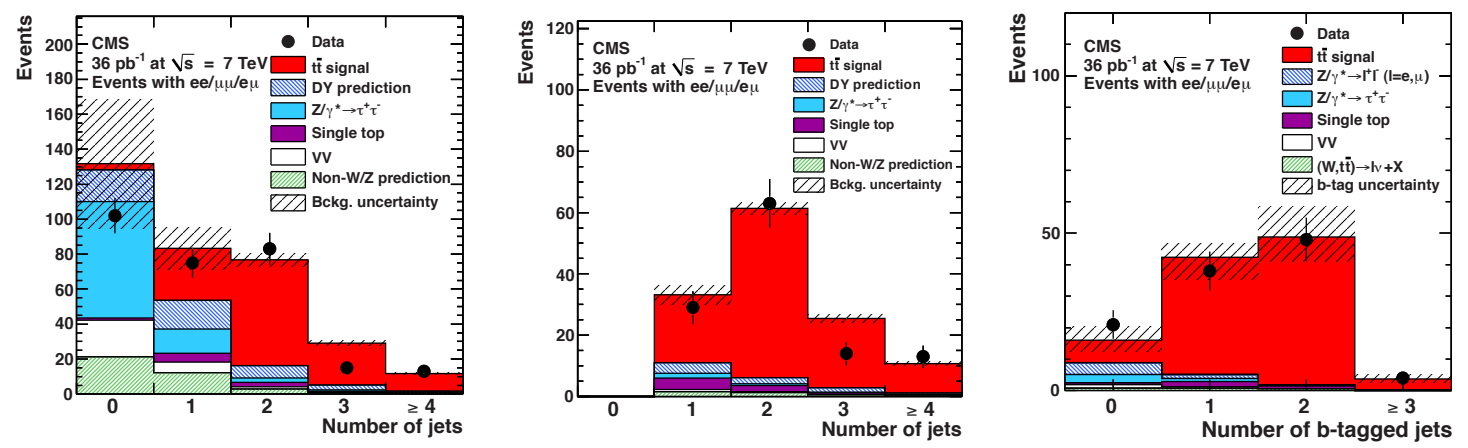

Figure 1: Dilepton sample distributions of the number of jets before (left) and after (middle) requiring at least one b-tagged jet in the event. The number of b-tagged jets for dilepton events is shown on the right.

Events in the dilepton channels are selected if there are two isolated leptons with transverse momentum $p_{t}$ larger than $20 \mathrm{GeV}$ (muons) and $30 \mathrm{GeV}$ (electrons). In addition, at least two jets with $p_{t}>30 \mathrm{GeV}$ are required of which at least one fulfills a $b$-tag signature. In the $\mu \mu$ and ee channels, dominant backgrounds from Drell-Yan processes are reduced by removing events in the region of the $Z^{0}$ resonance $\left(76<m_{\ell \ell}<106 \mathrm{GeV}\right)$ and requiring missing transverse energy $\not_{T}>30 \mathrm{GeV}$. The event sample obtained after all cuts is almost background free, as can be seen in figure 1 . The inclusive cross section is then determined from the counted number of events, taking into account remaining backgrounds and efficiencies. From the combination of the three dilepton channels a result of $\sigma_{t \bar{t}}=168 \pm 18$ (stat.) \pm 14 (syst.) \pm 7 (lumi.) pb is obtained [5], corresponding to a relative systematic uncertainty of about $9 \%$. The measurement agrees within errors with the theoretical predictions. 

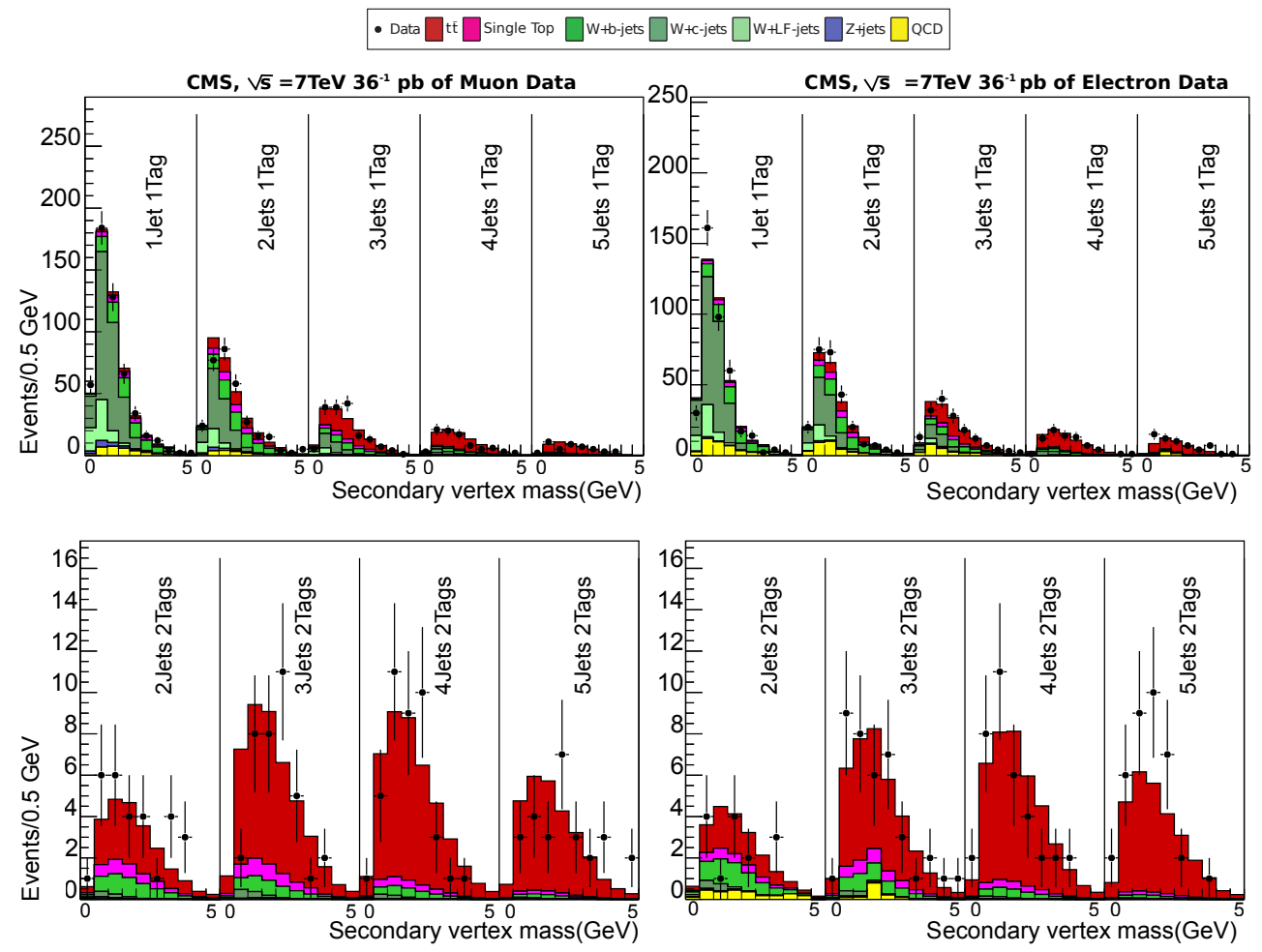

Figure 2: Result of the combined fit to the vertex mass distribution in different bins of jet multiplicity for 1 $b$-tag and $2 b$-tag samples in the $\mu+$ jet (left) and $e+$ jet (right) sample.

\section{Single Lepton-plus-Jets Channel}

In the channel with a single lepton (electron or muon) and jets, several independent measurements have been performed, requiring a single isolated high transverse momentum lepton and either three or more jets or the presence of a $b$-tag $[6,7]$. For the analysis with $b$-tag, a binned template fit to the invariant mass distribution of the tracks originating from the secondary vertex is performed in bins of the jet multiplicity (figure 2), simultaneously to the $\mu+$ jets and $e+$ jets samples. Systematic uncertainties, e.g. on the jet energy scale, the $b$-tag uncertainty and the background contribution from $W+$ jet events are taken into account in the fit. The measurement yields a cross section result of $\sigma_{t \bar{t}}=150 \pm 9$ (stat.) \pm 17 (syst.) \pm 6 (lumi.) pb, corresponding to a relative systematic error of $12 \%$. A measurement, independent of $b$-tagging, applies a simultaneous fit to the distributions of $E_{T}$ and the invariant mass of the three jets. The cross section result from this method is $\sigma_{t \bar{t}}=173_{-32}^{+39}$ (stat. + syst. $) \pm 7($ lumi. $) \mathrm{pb}$.

\section{Fully Hadronic Channel}

A first measurement of the $t \bar{t}$ production cross section is performed [9] using data recorded in 2011 with an integrated luminosity of $1.1 \mathrm{fb}^{-1}$. Selected events are required to have at least six high transverse momentum jets, including at least two jets with high-purity $b$-tag where the 


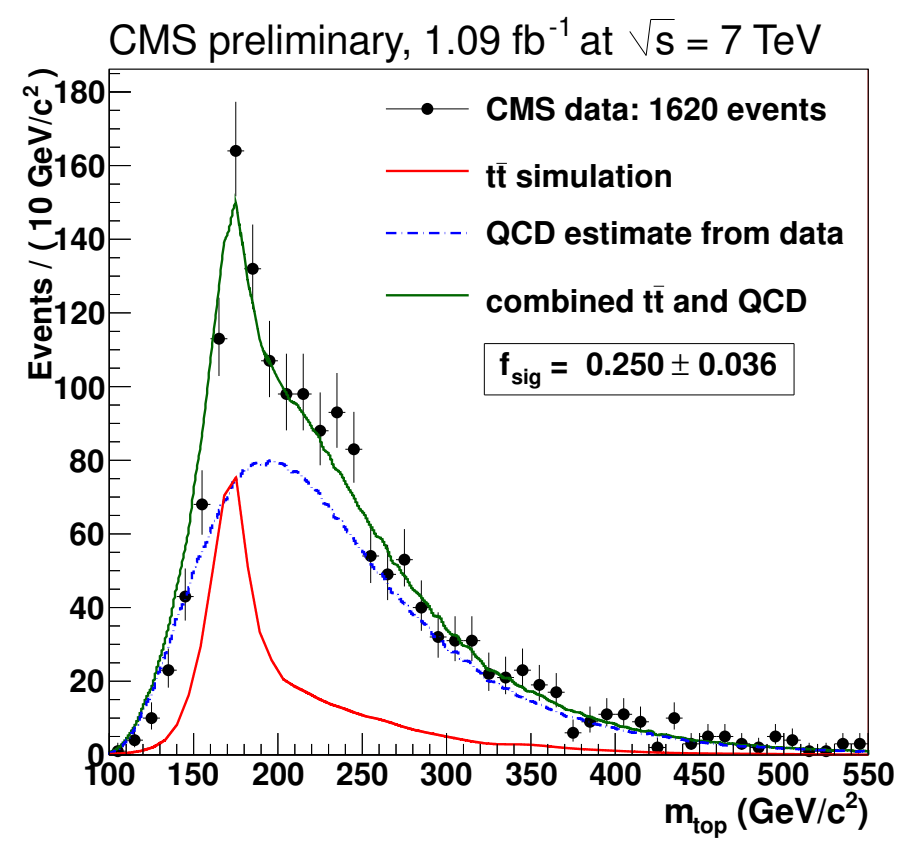

Figure 3: Result of the fit to the reconstructed top quark mass for the $t \bar{t}$ simulation (solid red line) and the multijet QCD estimated from data (dashed blue line).

secondary vertex containing at least three tracks is required to have a decay length significance of $d_{B}>2.0$. A kinematic reconstruction is performed on this event sample imposing constraints on the reconstructed $W$-mass and event-by-event equality of the reconstructed top and anti-top quark mass. In each event the one combination with minimal $\chi^{2}$ of the kinematic fit is selected. The relative fraction of $t \bar{t}$ signal, $f_{\text {sig }}=0.250 \pm 0.036$ (stat.), is determined from a template fit to the top quark mass distribution (figure4). The shape of the background is determined from the data. The measured cross section is $\sigma_{t \bar{t}}=136 \pm 20$ (stat.) \pm 40 (sys.) \pm 8 (lumi.) pb. The dominant uncertainties are the $b$-tagging, the jet energy scale and the QCD background.

\section{5. $\tau$-Lepton + Muon Channel}

Also, for the first time, a measurement in the channel $t \bar{t} \rightarrow W b W b \rightarrow \tau \nu_{\tau} b \mu_{v} \mu b$ is presented [8] using data with $1.1 \mathrm{fb}^{-1}$. Events are selected with exactly one isolated muon, and at least two jets of which at least one is required to have a $b$-tag. Non-t $t \bar{t}$ background events are further suppressed by rejecting events with $\not_{T}<40 \mathrm{GeV}$. Exactly one $\tau$ candidate is required with $p_{t}>20 \mathrm{GeV}$. The $\tau$ is identified as a narrow jet comprising a small number of charged hadrons or $\pi^{0}$, consistent with a decay from $\tau$. The photons from the $\pi^{0}$ decays are reconstructed in narrow $\eta-\phi$ strips in the electromagnetic calorimeter, taking into account possible conversions. The resulting event sample is presented in figure 5 left. The rate of jets, wrongly identified as $\tau$ is studied from the data and compared to the simulation (figure 5 right). The cross section, as obtained by counting the number of events above background and correcting for efficiencies and acceptance, yields $\sigma_{t \bar{t}}=148.7 \pm 23.6$ (stat. $) \pm 26.0$ (sys. $) \pm 8.9 \mathrm{pb}$. 

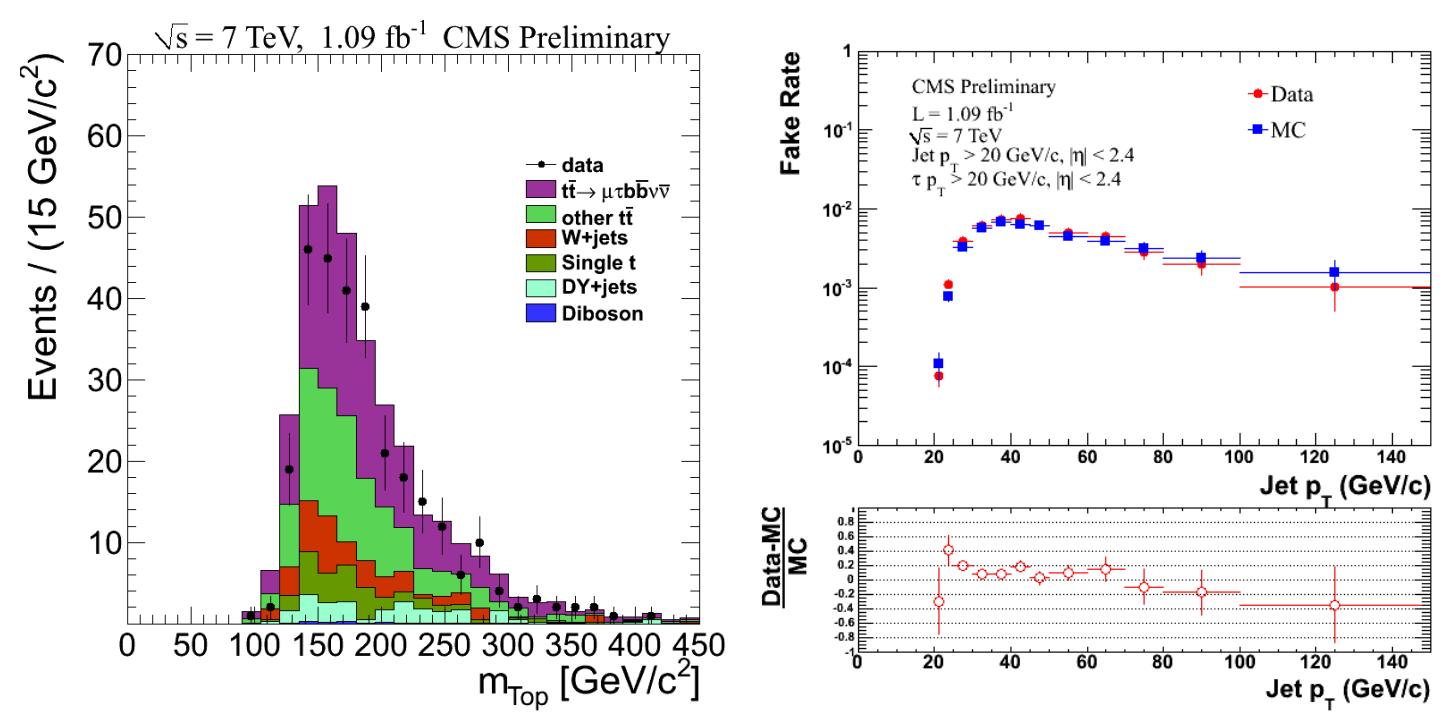

Figure 4: Left: Reconstructed top quark mass distribution for the tau dilepton candidate events. Right: Tau fake rate distribution from QCD multijet samples.

\section{Summary}

In conclusion, a full set of measurements of $t \bar{t}$ production cross sections have been presented. In the dilepton and lepton+jet channels the results are approaching systematic limitations and the experimental precision is starting to constrain theory predictions. First results in two previously unmeasured decay channels, the fully hadron channel and the $\tau+\mu$ channel, have also been presented. All the results are in agreement with each other and with predictions from QCD calculations.

\section{References}

[1] CMS Collaboration, 'The CMS experiment at the CERN LHC', JINST 3:S08004,2008.

[2] N. Kidonakis, Phys.Rev. D82 (2010) 114030.

[3] U. Langenfeld, S. Moch and P. Uwer, Phys. Rev. D 80 (2009) 054009; M. Aliev, H. Lacker, U. Langenfeld et al., Comput.Phys.Commun. 182 (2011) 1034âĂŞ1046.

[4] V. Ahrens, A. Ferroglia, M. Neubert, B.D. Pecjak and L.L. Yang, JHEP 09 (2010) 097.

[5] S. Chatrchyan et al. [CMS Collaboration], JHEP 1107 (2011) 049.

[6] S. Chatrchyan et al. [CMS Collaboration], Eur. Phys. J. C 71 (2011) 1721.

[7] S. Chatrchyan et al. [CMS Collaboration], arXiv:1108.3773 [hep-ex], accepted by PRD.

[8] CMS Collaboration, CMS-PAS-TOP-11-006, 2011.

[9] CMS Collaboration, CMS-PAS-TOP-11-007, 2011. 